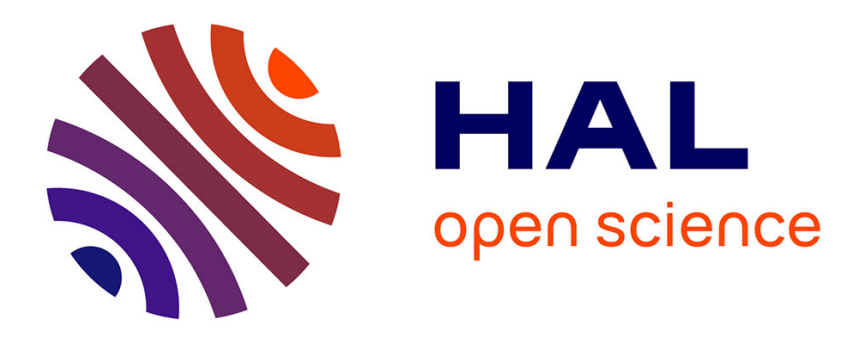

\title{
Politiques d'emploi et territoires (Employment policies and territories)
}

Raymonde Séchet

\section{To cite this version:}

Raymonde Séchet. Politiques d'emploi et territoires (Employment policies and territories). Bulletin de l'Association de géographes français, 2000, Emploi et chômage, 77 (3), pp.238 - 251. 10.3406/bagf.2000.2169 . halshs-01438760

\section{HAL Id: halshs-01438760 https://shs.hal.science/halshs-01438760}

Submitted on 18 Jan 2017

HAL is a multi-disciplinary open access archive for the deposit and dissemination of scientific research documents, whether they are published or not. The documents may come from teaching and research institutions in France or abroad, or from public or private research centers.
L'archive ouverte pluridisciplinaire HAL, est destinée au dépôt et à la diffusion de documents scientifiques de niveau recherche, publiés ou non, émanant des établissements d'enseignement et de recherche français ou étrangers, des laboratoires publics ou privés. 


\section{Politiques d'emploi et territoires (Employment policies and} territories)

Raymonde Séchet

\section{Citer ce document / Cite this document :}

Séchet Raymonde. Politiques d'emploi et territoires (Employment policies and territories). In: Bulletin de l'Association de géographes français, 77e année, 2000-3 ( septembre). Emploi et chômage. Taïwan. Alger. pp. 238-251;

doi : 10.3406/bagf.2000.2169

http://www.persee.fr/doc/bagf_0004-5322_2000_num_77_3_2169

Document généré le 19/04/2016 


\title{
Résumé
}

Résumé. - De la diversification des acteurs impliqués dans la lutte contre le chômage et de l'évolution des formes d'intervention, découle une géographie des politiques de l'emploi marquée par la complexité : inégalement répartie dans l'espace, plus importante et plus variée en milieu urbain, l'offre d'insertion par l'économique est le reflet des structures économiques locales et de l'inégale mobilisation des acteurs ; l'insertion a créé ses propres découpages qu'il s'agit aujourd'hui de mettre en cohérence et d'adapter aux besoins d'une gestion de proximité ; par les partenariats sur lesquels ils reposent, les dispositifs actuels sont créateurs de territoires. Les projets de développement social local qui sont pilotés au nom de la citoyenneté comportent aussi des risques de repli sur l'espace de proximité, et donc d'exclusion.

\begin{abstract}
Abstract. - The massive growth of unemployment has been mobilising more and more forces and the local communities are becoming increasingly involved in professional integration schemes. Owing to the increasing variety of the bodies involved and to the evolution of their modes of action, the geography of integration and employment policies is characterised by its complexity ; with their uneven geographical distribution - they are more numerous and diverse in urban areas - the opportunities for professional integration provided by the economic forces reflect the local economic structures and the varying degree of mobilisation of the social forces. The policy of integration has thus generated its own boundaries, which must now be brought into line with each other and adapted to the needs of local management. Because of the partnerships they rely on, the current systems are creating new territories. The projects of local, social development that are conducted for the sake of citizenship also present risks of withdrawal into one's own local area and, therefore, of social exclusion.
\end{abstract}


Bull. Assoc. Géogr. Franç., 2000 - 3

\title{
Raymonde SÉCHET* \\ POLITIQUES D'EMPLOI ET TERRITOIRES
}

\author{
(EMPLOYMENT POLICIES AND TERRITORIES)
}

\begin{abstract}
RÉSUMÉ. - De la diversification des acteurs impliqués dans la lutte contre le chômage et de l'évolution des formes d'intervention, découle une géographie des politiques de l'emploi marquée par la complexité: inégalement répartie dans l'espace, plus importante et plus variée en milieu urbain, l'offre d'insertion par l'économique est le reflet des structures économiques locales et de l'inégale mobilisation des acteurs; l'insertion a créé ses propres découpages qu'il s'agit aujourd'hui de mettre en cohérence et d'adapter aux besoins d'une gestion de proximité; par les partenariats sur lesquels ils reposent, les dispositifs actuels sont créateurs de territoires. Les projets de développement social local qui sont pilotés au nom de la citoyenneté comportent aussi des risques de repli sur l'espace de proximité, et donc d'exclusion.
\end{abstract}

Mots-clés: chômage, insertion, politiques territoriales, partenariat, Rennes, Ille-et-Vilaine.

\begin{abstract}
The massive growth of unemployment has been mobilising more and more forces and the local communities are becoming increasingly involved in professional integration schemes. Owing to the increasing variety of the bodies involved and to the evolution of their modes of action, the geography of integration and employment policies is characterised by its complexity; with their uneven geographical distribution - they are more numerous and diverse in urban areas - the opportunities for professional integration provided by the economic forces reflect the local economic structures and the varying degree of mobilisation of the social forces. The policy of integration has thus generated its own boundaries, which must now be brought into line with each other and adapted to the needs of local management. Because of the partnerships they rely on, the current systems are creating new territories. The projects of local, social development that are conducted for the sake of citizenship also present risks of withdrawal into one's own local area and, therefore, of social exclusion.
\end{abstract}

Key words: unemployment, professional integration, regional development policies, partnership, Rennes, Ille-et-Vilaine.

L'idée de politique de l'emploi émerge dans les années soixante lorsque l'économie doit faire face à d'importantes restructurations et à des suppressions d'emplois (mines, constructions navales, sidérurgie) alors que les branches en plein essor (automobile, électronique) satisfont

* RESO - UMR 6590, Université Rennes 2. 
difficilement leurs besoins en main-d'œuvre. L'objectif est de réduire le chômage frictionnel en favorisant la mobilité sectorielle et géographique de la main-d'œuvre (création de l'AFPA en 1966 et de l'ANPE en 1967). Le chômage, pas encore objet géographique, n'est étudié qu'accessoirement par la géographie économique et régionale (Montagné-Villette, 1999). Pierre Estienne, qui n'aborde l'emploi que par les difficultés de recrutement des entreprises, écrit encore en 1979 à propos des charbonnages du nord: "On passe de 200000 emplois dans les houillères en 1946 à 41000 en 1976, les effectifs du fond tombant dans le même temps de 135000 à environ 19000; une main-d'œuvre qu'il avait fallu faire venir à grands frais et loger en faisant sortir du sol des villes entières. [...] Et les familles de mineurs où on travaillait au fond depuis plusieurs générations ont si bien ressenti la crise que les Houillères n'arrivent même pas à recruter le personnel qui leur est encore nécessaire et que la part de la main-d'œuvre nord-africaine, surtout marocaine, ne cesse d'augmenter " (Estienne, 1979, p. 154).

Sous l'effet de sa progression notable à partir de 1974, de son maintien à un niveau élevé et de l'allongement de ses durées moyennes, le chômage n'est plus seulement pensé comme un problème strictement économique mais aussi comme une réalité sociale à traiter pour elle-même. Tout en révélant l'inefficacité des politiques macro-économiques, la massification du chômage impose la mise en œuvre de dispositifs conçus pour en atténuer les conséquences sociales autant que pour améliorer réellement la situation de l'emploi. Dès lors, "politique de l'emploi " va s'écrire au pluriel (Join-Lambert, 1994, p. 181). Les différents dispositifs s'organisent selon une distinction entre dépenses passives et dépenses actives: les premières visent à apporter un revenu aux chômeurs en les indemnisant et à assurer la solidarité entre générations par des incitations financières à sortir de la population active (préretraites, aides au retour); très variées - mesures d'aide à l'embauche, à la création d'emplois et d'entreprises, à la formation pour améliorer l'employabilité, etc.) -, les secondes cherchent à éviter le chômage d'exclusion, à faciliter l'insertion ou la réinsertion, et prennent pour cela une forme de plus en plus territorialisée.

Bien que l'Etat demeure le principal acteur public de la lutte contre le chômage, les collectivités locales y sont toujours plus impliquées; les instances de concertation, de gestion et d'observation se sont multipliées. De cette diversification des acteurs et de l'évolution des formes d'intervention vers davantage de territorialisation, découle une géographie de l'insertion et des politiques de l'emploi marquée par la complexité: inégalement répartie dans l'espace, et notamment plus importante et plus variée en milieu urbain, l'offre d'insertion par l'économique reflète les structures économiques locales et l'inégale mobilisation des acteurs; l'insertion a créé ses propres découpages qu'il s'agit aujourd'hui de mettre en cohérence et $d^{\prime}$ adapter aux besoins d'une gestion de proximité; par les partenariats sur lesquels ils reposent, les dispositifs actuels sont créateurs de territoires. Notre propos, relativement général, s'appuie sur de nombreuses études de terrain conduites en Ille-et-Vilaine. 


\section{L'offre d'insertion pour les chômeurs de longue durée}

Les chômeurs de longue durée sont devenus la priorité des politiques de l'emploi dans la seconde moitié des années quatre-vingt. Pour ces "publics en grandes difficultés", deux grands types d'actions ont été privilégiés: des stages de formation pour agir sur l'employabilité et des contrats de travail spécifiques relevant surtout du secteur non-marchand et de l'insertion par l'économique. Instaurés en 1990, les contrats emploisolidarité (CES) peuvent être signés par des collectivités territoriales, des établissements publics, des associations. Le dispositif a été complété en 1992 par les contrats emplois consolidés pour les personnes sans emploi à l'issue de leur CES. Les emplois-jeunes constituent aujourd'hui le troisième type important de contrats du secteur non-marchand. Dans les trois cas, la localisation des offres dépend de celles des signataires potentiels.

\section{- Les atouts des villes}

Les logiques de polarisation et de concentration des activités de services dans les grandes villes s'imposent aux activités d'insertion. Ainsi, en janvier 1995, Rennes regroupe $46 \%$ des employeurs de Contrats emploisolidarité dans la zone d'emploi de Rennes et toutes les communes du district embauchent des personnes en contrats emploi-solidarité contre seulement $54 \%$ des communes de la zone d'emploi hors district (Eydoux, 1995). A cet avantage quantitatif, la grande ville ajoute l'atout qualitatif, c'est-à-dire la diversité de l'offre d'activités d'insertion tant dans les structures d'insertion par l'économique que dans les activités de type social ou culturel (en janvier 1995, les organismes rennais qui disposent des plus gros effectifs de CES sont la Ville de Rennes [172], le Conseil général [134], le Centre hospitalier régional [109] et le Centre hospitalier spécialisé [84]; I'Université Rennes 2 compense son sous-encadrement en personnel IATOS [279 postes] par le recrutement de plus de $100 \mathrm{CES}$ ). Dans les communes hors district, les principaux employeurs sont les mairies et les écoles primaires pour des activités d'entretien des locaux, de la voirie, des espaces verts ou d'espaces naturels: les mairies représentent $47 \%$ des employeurs de personnes en Contrats emploi-solidarité dans les communes de la zone d'emploi de Rennes hors district contre seulement $10 \%$ dans les communes du district.

\section{- Les «nouveaux services", signe de vitalité des campagnes}

Cette concentration urbaine de l'offre d'insertion peut paraître répondre à l'inégale densité des besoins. Les situations de chômage de longue durée n'en sont pas moins réelles en milieu rural où les acteurs de l'insertion sont, comme ceux des villes, confrontés au chômage et aux difficultés 
d'insertion des jeunes. D'autant plus que les dispositifs mis en œuvre dans le cadre de la politique du logement des plus démunis peuvent donner lieu à des mobilités de la ville vers la campagne. Les logements proposés à des ménages aux ressources limitées et peu mobiles, soit par absence de moyens de locomotion, soit parce que les déplacements sont coûteux, sont souvent éloignés des circuits de transports en commun, et surtout des zones principales d'emplois.

L'accueil de nouvelles populations, de manière durable pour les nouveaux résidents, surtout nombreux dans les couronnes périurbaines ou de manière temporaire pour les activités de loisirs, est favorable à l'essor des activités d'insertion. La présence de nouveaux résidents oblige les collectivités locales à réfléchir à une offre croissante de services de proximité. Liés à l'évolution des modes de vie entraînée par la double activité dans les ménages, ces besoins sont variés: garde d'enfants, activités culturelles et de loisirs, entretien de la maison et du jardin...

Pour fixer des populations en quête d'une nature idéalisée, il faut préserver les qualités que recèlent ces espaces. Tout un ensemble de services de proximité ont pour mission de valoriser, protéger et entretenir les espaces naturels et bâtis. Les salariés des chantiers d'insertion, souvent appelés "brigades vertes", effectuent des travaux de préservation et de mise en valeur du paysage (chemins et talus bocagers, cours d'eau et étangs, etc.). Dans le cadre de CES, bénéficiaires du RMI et demandeurs d'emploi de longue durée nettoient, débroussaillent, élaguent, fleurissent, aménagent des chemins de randonnées et des aires de pique-nique.

Qu'elles aient pour objectif de maintenir une vie sociale intense, de prendre en charge des services à la population, d'améliorer le cadre de vie et l'environnement, des possibilités d'activités d'insertion existent donc en milieu rural. Pour des raisons d'efficacité et de partage de la charge financière, les initiatives s'appuient fréquemment sur des dispositifs intercommunaux et sur le dynamisme des associations. Par exemple, à l'exception de la ferme d'insertion de Parcé, l'intercommunalité apparaît dans tous les dispositifs d'insertion en milieu rural de la zone d'emploi de Fougères (Hervé, 1996). C'est aussi dans l'intercommunalité que peut s'organiser la mise en œuvre de transports collectifs afin d'accroître les mobilités vers l'emploi et que peuvent être envisagées des solutions de proximité pour résoudre les problèmes de garde des jeunes enfants qui limitent l'accès des femmes à l'emploi. Malgré tout, contrairement aux grandes villes où les tâches administratives représentent une part importante des CES, les activités proposées en milieu rural (entretien de voiries, espaces verts et naturels) s'adressent surtout à des publics masculins.

\section{Les politiques d'emploi : un dispositif complexe}

La géographie de l'offre dépend des caractéristiques économiques mais aussi de la mobilisation des acteurs locaux, qu'ils soient politiques ou associatifs, pour mettre en œuvre les dispositifs prévus par l'Etat. La loca- 
lisation des politiques d'insertion, c'est-à-dire l'application de décisions nationales en tenant compte des modes d'organisation et caractéristiques de la zone d'intervention qui sert de cadre à cette action, s'appuie sur un partage des compétences et sur des découpages qui nuisent à leur visibilité, et donc, sans doute aussi, à leur efficacité.

\section{- Diversité des acteurs}

Le colloque "Une stratégie locale pour l'emploi " organisé en février 1983 par le ministère de l'Emploi a officiellement consacré l'irruption du local dans le champ des politiques de l'emploi (Wuhl, 1996). II s'agit d'améliorer le fonctionnement du marché du travail par la mobilisation des initiatives de terrain et la recherche de l'adaptation de la main-d'œuvre aux nouvelles exigences des entreprises. Dans la seconde moitié des années 1980, la faible sensibilité du chômage de longue durée aux effets de la reprise de l'emploi et l'importance du chômage récurrent des jeunes actifs favorisent l'émergence de la notion de chômage d'exclusion et, donc, l'évolution des politiques vers un meilleur ancrage dans le milieu local.

Les élus des différentes circonscriptions territoriales sont tous, peu ou prou, par délégation de compétence ou par choix et contrainte du moment, mobilisés en faveur de l'insertion. L'action pour l'emploi implique aussi de multiples acteurs en charge de gestion. Pour assurer le fonctionnement du marché du travail, de la politique de lutte contre le chômage, et plus largement de la lutte contre l'exclusion, l'administration est présente au plan local à travers ses directeurs du travail et de l'emploi, ses directeurs de I'ANPE, ses coordonnateurs emploi-formation, ses commissaires à l'emploi. A ces multiples représentants de I'Etat, il faut ajouter des acteurs déconcentrés tels que les ASSEDIC, mais aussi divers dispositifs mis en place à partir de 1982 dans le cadre de l'action en faveur de l'insertion: Missions locales, Programmes locaux d'insertion par l'économique (PLIE), Commissions locales d'insertion (CLI), Commissions locales de I'habitat (CLH) qui, par leur intervention dans l'insertion par I'habitat, peuvent contribuer indirectement à la réinsertion dans l'économie.

On pourrait espérer qu'une grande cohérence organisationnelle au niveau local vienne corriger les risques inhérents au partage des compétences et à la complexité des dispositifs en matière d'insertion et de lutte contre le chômage. II n'en est rien.

\section{- Incohérence des découpages}

Bien que devant en principe répondre au devoir d'égalité d'accessibilité entre citoyens, les mailles conçues pour mettre en œuvre les politiques de l'emploi et de l'insertion résultent en réalité d'arbitrages entre recherche de l'efficacité gestionnaire, commodité par adéquation avec des découpages déjà existants, visées électoralistes, etc. L'exemple du département 
de l'llle-et-Vilaine, pourtant souvent considéré comme exemplaire, suggère que l'action s'est faite sans qu'il $y$ ait eu recherche de cohérence (Séchet et Le Délézir, 1998).

Les aires d'intervention des antennes ASSEDIC et des agences locales pour I'emploi (ALE) (carte 1B) ne se superposent que pour les ALE et les antennes ASSEDIC de Fougères et de Vitré. Au nord, I'ALE de Saint-Malo épouse les limites de l'arrondissement alors que l'antenne ASSEDIC s'arrête aux limites de la zone d'emploi (carte 1A). L'ALE de Redon s'étend sur trois départements et deux régions (les cantons morbihannais d'Allaire et de La Gacilly et le canton de Saint-Nicolas de Redon en Loire-Atlantique en dépendent). Alors que l'ANPE est un des principaux partenaires impliqués dans le fonctionnement des Missions locales, les aires d'intervention des ALE et des Missions locales ne se superposent que pour Saint-Malo et Fougères (carte 1C). La Mission locale de Vitré s'étend sur l'ALE de Vitré et sur les cantons de Janzé et Retiers dépendant de I'ALE de Rennes-Poterie. La Mission locale de Redon recoupe les aires d'intervention des ALE de Redon et de Rennes-Poterie, ainsi que celles de Vannes et de Chateaubriant en Loire-Atlantique. L'aire d'intervention de la Mission locale de Rennes ne correspond ni aux découpages des ALE ni aux limites de la zone ASSEDIC, ni d'ailleurs à celles du Comité de développement économique et social du Pays de Rennes (CODESPAR).

Les textes de mise en application du RMI prévoyaient une délimitation des CLI selon des aires cohérentes sur les plans économique et social plutôt qu'en rapport avec des découpages administratifs. Dans la réalité, l'llle-et-Vilaine n'a pas échappé aux arbitrages entre logique fonctionnelle et logique gestionnaire de délimitation en fonction des circonscriptions d'action sociale ou des limites d'arrondissements. Alors que la CLI de Vitré empiète sur deux ALE, les CLI de Fougères et du Pays malouin ont été calquées sur les limites d'arrondissements et débordent sur la zone d'emploi de Rennes (carte 1D). Dans la mesure où le département est le cadre de mise en œuvre du RMI, la $\mathrm{CLI}$ de Redon ne pouvait pas coïncider avec l'ALE. De même, la délimitation des CLH, qui pouvaient être organisées sur la base des arrondissements, des circonscriptions d'action sociale, des CLI, mais aussi en fonction des bassins d'habitat, illustre bien le pouvoir d'intervention de certains acteurs locaux: I'hypothèse première lors de la préparation du Plan départemental d'action pour le logement des personnes défavorisées, qui était de faire coïncider les CLH avec les CLI, a d'emblée été battue en brèche par le District de Rennes.

Le partage des compétences et l'accumulation d'aires d'intervention qui ne se superposent pas rendent l'ensemble du dispositif de lutte contre l'exclusion compliqué et peu lisible. Autour de chaque ville importante du département se dessinent des "centres" qui correspondent aux aires de recouvrement des antennes ASSEDIC, ALE, Missions locales, CLI, CLH. La confrontation entre les différents découpages révèle tout aussi clairement l'existence de "marges" qui peuvent être rattachées à plusieurs villes de gestion. On peut faire la double hypothèse que ces aires de non-recouvrement sont moins bien connues des institutions faute de ces échanges et circulations d'informations qui permettent une vision globale des territoires, et que, d'autre part, les habitants peuvent y rencontrer des difficultés à accéder au bon guichet. 
Réalités fonctionnelles et découpages: Zones d'emploi et ALE en Ille et Vilaine.

A

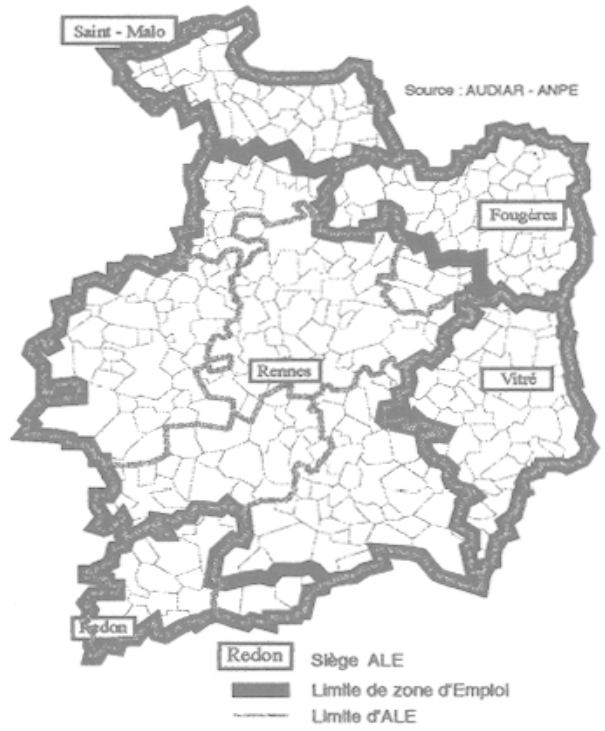

Espaces d'intervention

de l'ANPE et des Missions locales en Ille-et-Vilaine

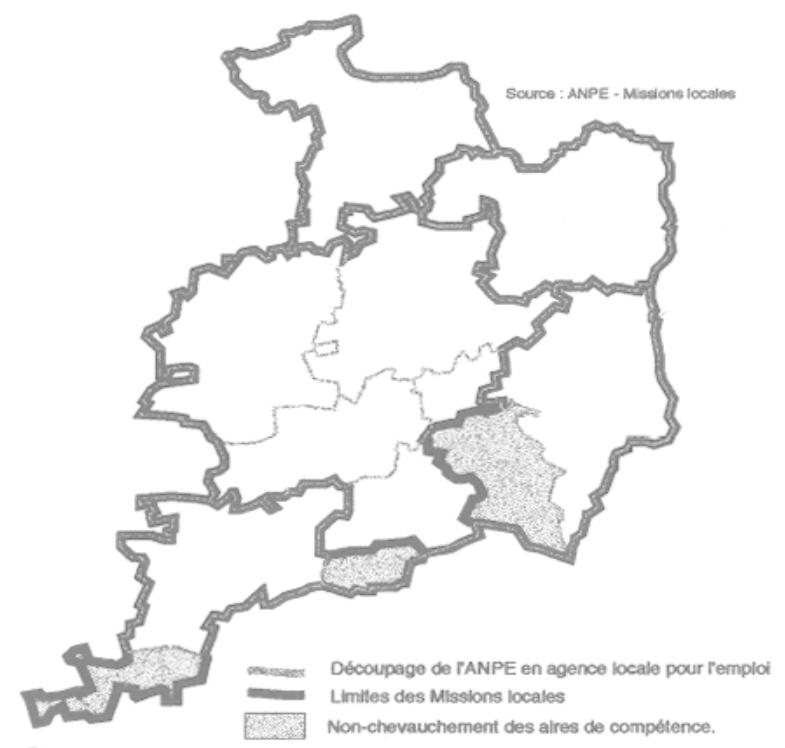

C
Espaces d'intervention
de l'ANPE et de l'ASSEDIC en Ille-et-Vilaine

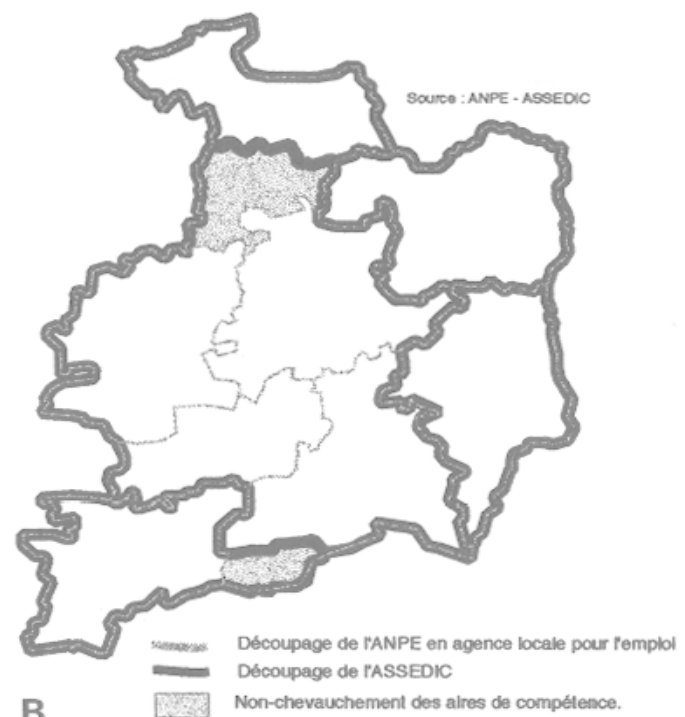

Espaces d'intervention

de l'ANPE et CLI en Ille-et-Vilaine

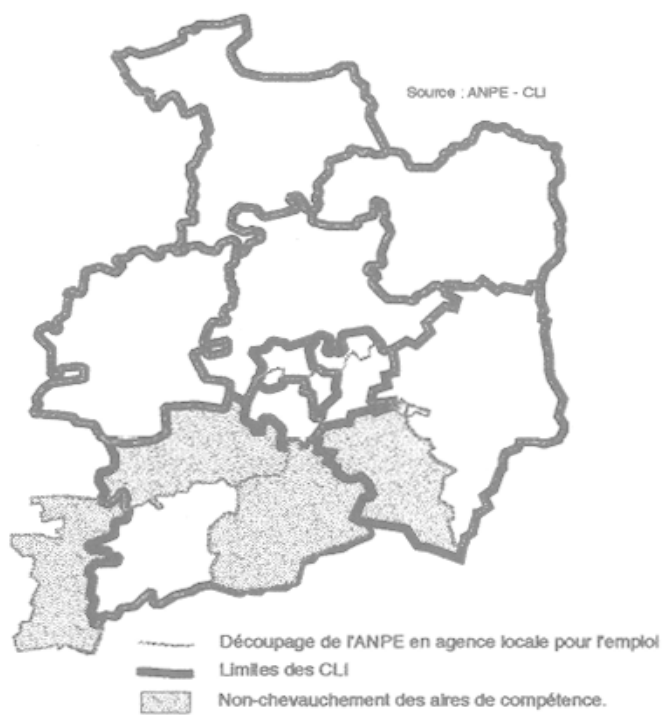

D

Conception cartographiqua : Le Carour E - Le Detazir R. - Howain S. - Stchot R.

Carte 1. Les découpages de l'insertion en Ille-et-Vilaine.

\section{- Du maillage au tissage de l'espace}

Pour Jean-Louis Guigou, le "pays", pensé comme "lieu d'expression d'une communauté d'intérêts économiques et sociaux et de solidarités réciproques entre la ville et l'espace rural", s'impose dans la mesure où 
des découpages différents compliquent largement, voire obèrent toute idée de partenariat autour de la lutte contre l'exclusion et laisseraient les espaces ruraux à l'écart de la mobilisation pour l'emploi (Guigou, 1995). Un référent territorial unique et stable déboucherait sur une meilleure efficacité. Le gain serait direct par une amélioration de la connaissance de l'espace et de ses habitants, de la qualité des diagnostics et des évaluations. Les orientations nationales pourraient ainsi être mises en œuvre de manière plus proche des réalités locales. II serait aussi indirect grâce à une meilleure lisibilité des projets et actions menées par l'Etat. Cette maille unique pourrait ne pas être seulement cadre d'intervention localisée et déconcentrée, mais aussi espace où l'habitude prise par les différents acteurs de travailler ensemble permettrait le croisement de politiques descendantes et ascendantes dans une perspective de développement économique et social local.

La question des bases sur lesquelles repenser des découpages unifiés est grosso modo une alternative entre circonscriptions administratives et institutionnelles déjà existantes et espaces fonctionnels: I'ajustement entre mailles du système administratif et territoires vécus devrait permettre de corriger les dysfonctionnements nés de l'inadéquation croissante entre des territoires vécus évolutifs et des mailles administratives héritées et figées; mais c'est précisément parce que les territoires vécus évoluent au rythme des migrations que cette recherche de l'adéquation entre territoires fonctionnels et territoires institutionnels peut relever de la course sans fin (Maurel et Lefèvre, 1995). Le débat, classique en géographie, entre région fonctionnelle et espaces vécus, a clairement démontré les limites d'une approche par les pratiques spatiales: les bassins de vie et les zones d'emploi sont des représentations statistiques produites sur la base des comportements dominants mesurés par des chiffres de flux et qui ne sont pas ceux des populations concernées par les politiques de l'emploi et de l'insertion. Les espaces de l'insertion ne sont pas les mêmes que ceux des marchés locaux du travail. Les découpages conçus pour animer les politiques de l'emploi peuvent-ils permettre de concilier le niveau de la cohésion sociale, c'est-à-dire l'insertion de proximité, avec un niveau plus large et que ses promoteurs imaginent comme espace de projet, de développement et d'efficacité économique (Guigou, 1996)?

Au total, l'évidente nécessité d'harmoniser les découpages n'est qu'apparente. Le souci d'inventer un découpage unique ne doit pas occulter les réflexions sur le contenu des mailles et les modalités de leur fonctionnement. Les incohérences observées en Ille-et-Vilaine peuvent, si elles sont analysées comme le reflet de la mobilisation des acteurs locaux, être interprétées de manière positive comme des preuves de dynamisme territorial (Augustin et al., 1991). La confusion des découpages autour de Redon tient à un effort de mise en adéquation avec les territoires fonctionnels. Cependant les modalités du partage des compétences - base départementale du RMI, organisation régionale de la formation professionnelle et des ASSEDIC par exemple - et la multiplicité des acteurs créent alors de nouvelles difficultés: le Pays de Redon et de Vilaine est partagé entre quatre arrondissements, cinq circonscriptions législatives, cinq CLI, six 
ALE, cinq antennes ASSEDIC! La carte des CLH en Ille-et-Vilaine découle, quant à elle, de l'effort en matière de droit au logement mené dans le cadre du District de Rennes. En fait, l'ensemble des dispositifs d'insertion bénéficie de la forte mobilisation rennaise. Créés respectivement en 1984 à l'échelle du bassin d'emploi et en 1993 au niveau du district de Rennes, le CODESPAR et le PLIE sont certes venus ajouter de la complexité organisationnelle, mais ils ont permis d'améliorer la connaissance des besoins (création d'un observatoire de l'emploi) et de considérablement étoffer l'offre d'insertion (soutiens à la création d'associations intermédiaires, d'entreprises d'insertion...). Au risque de renforcer les inégalités entre Rennes et les espaces ruraux de sa périphérie.

Pour des raisons qui ne sont pas seulement matérielles, des personnes, souvent en milieu rural, ont une approche spécifique du marché du travail en ce sens où, dans leur quête d'emploi, la distance par rapport au domicile passe avant la nature de l'activité. Les objectifs de nature économique ne doivent pas faire oublier les besoins d'insertion de proximité des personnes qui sont restées à l'écart des formes modernes de mobilité et dont les bassins d'emploi personnel sont singulièrement étroits. Pour cela le pays, niveau du projet économique, doit s'appuyer sur les regroupements intercommunaux, c'est-à-dire sur le bassin de vie, "niveau de gestion écologique et sociale des rapports de voisinage" (Guigou, 1995), et donc niveau de la cohésion sociale.

Diverses expériences en matière de déconcentration des services et de modes d'intervention relèvent d'une stratégie d'émergence de relais au contact de la population pour favoriser l'accès à l'information. En 1992, le CODESPAR a proposé d'organiser dans la proximité l'accueil des demandeurs d'emploi par la mise en place dans plusieurs communes du bassin d'emploi de Rennes de "points relais" en collaboration avec l'ANPE, les Centres d'information et d'orientation, la Mission locale. La clarification des conditions du partenariat entre l'ANPE et le Conseil Général en 1994 a permis d'étendre l'accueil et l'information de proximité dans les secteurs les plus ruraux du département. Aujourd'hui, même si des disparités subsistent en faveur du bassin de Rennes, des Points Accueil-Emploi (Rolland, 1999) ont été ouverts sur l'ensemble du département (carte 2), souvent dans le cadre des communautés de communes. Ils interviennent dans les domaines de l'emploi, de l'insertion, de la formation, en articulation avec la politique locale de services de proximité. Ces déconcentrations nécessitent la volonté des communes ou communautés de communes, et parce que les services de proximité ont un coût, et parce qu'elles supposent un décloisonnement des compétences sur la base du principe du guichet unique, ce qui implique que soient surmontées des résistances catégorielles.

Cette redéfinition de la rencontre entre de l'organisationnel horizontal et de l'administratif vertical est un préalable à une réelle articulation de l'échelon de la décision avec les bourgs, les villages, les quartiers. L'ensemble pourrait alors déboucher sur la conception au niveau local d'un "système d'insertion" capable d'établir la cohérence des politiques d'insertion, la bonne définition de l'espace local étant "celle qui permet au plus grand nombre de partenaires de construire un projet fédérateur, sus- 


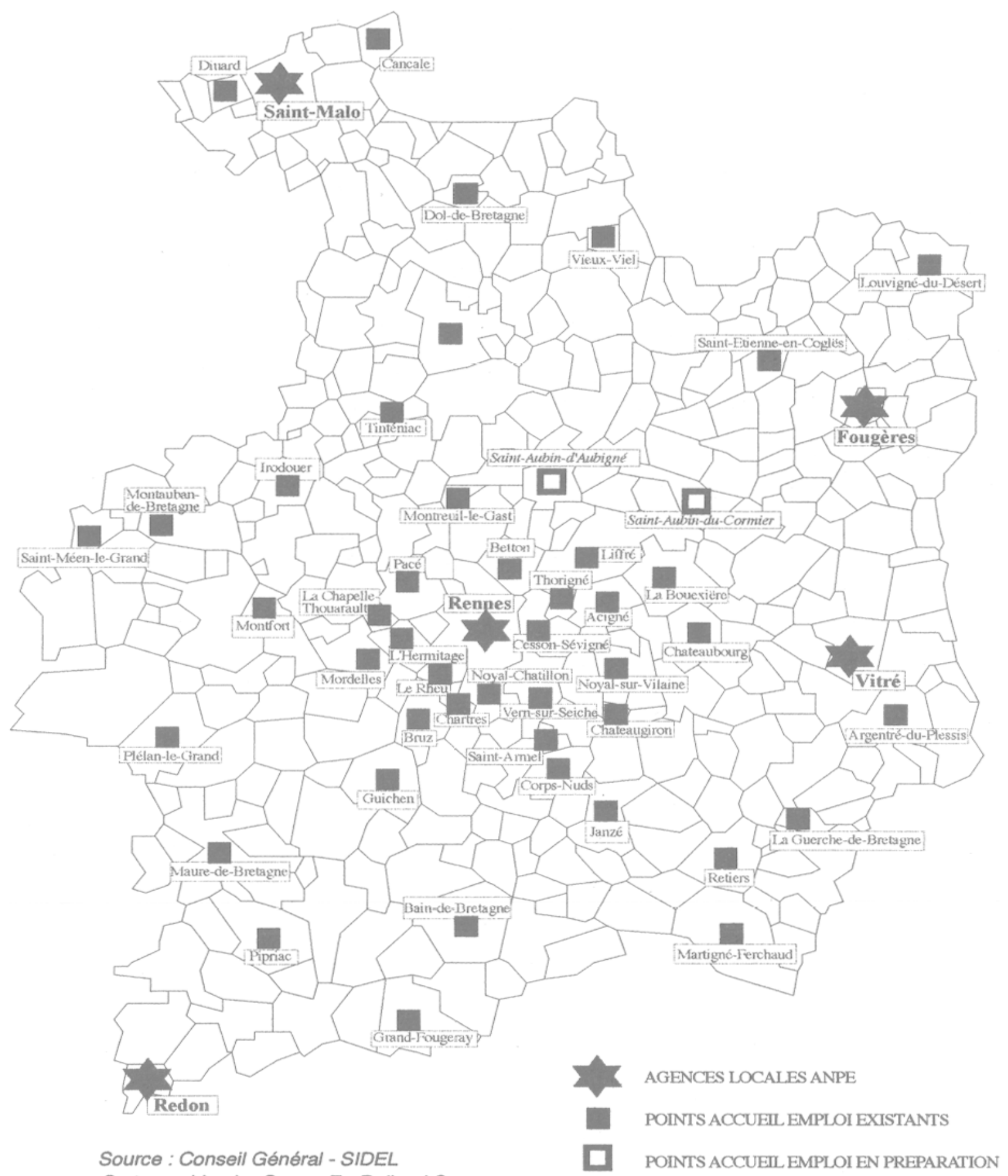

Cartographie : Le Carour E.- Rolland S.

Carte 2. Points accueil emploi.

Situation en décembre 1998.

ceptible d'aborder les problèmes de l'exclusion dans une démarche globale, et autant que possible intégrée dans un projet de développement local » (Le Carpentier, 1992). 


\section{Des politiques de l'emploi créatrices de territoires}

Outrepassant leur incompétence de droit, les communes et les différentes collectivités locales ont progressivement élargi leur implication d'une simple attention portée au développement économique vers l'ensemble des politiques d'emploi. La lutte contre l'exclusion tient une place importante dans les projets de pays et les Chartes de territoires dont ils se dotent actuellement. Le Pays de Brocéliande en Ille-et-Vilaine qui illustre bien le passage d'une stricte logique économique à une lecture plus globale et sociale du développement a gagné en reconnaissance sous l'effet d'une mobilisation de tous ses acteurs en faveur de l'insertion par l'économique. Entre 1994 (convention cadre de développement signée en parallèle au Contrat de Plan) et aujourd'hui, les priorités sont passées de une (la valorisation du potentiel agro-industriel) à quatre (la mobilisation pour l'emploi et contre l'exclusion; la valorisation des ressources locales et le renforcement des services par la mise en réseaux; l'organisation du territoire pour un développement durable; l'affirmation du pays par sa promotion et la mise en commun des compétences).

Pour les maires, l'insertion sociale et professionnelle requiert une gestion de proximité à la fois individualisée et globale: "Pour mieux appréhender les besoins des demandeurs d'emploi ou des personnes en situation de précarité et d'exclusion, assurer la cohérence des actions et la coordination de tous les acteurs, conjuguer développement économique et solidarité, il convient de passer d'une logique de dispositifs et guichets à une stratégie de projet, d'ancrer la politique de l'emploi sur le territoire" (AMF, 1997). Si elle peut prendre des formes très différentes, cette dynamique d'ancrage dans le tissu local répond toujours au même enjeu: l'efficacité de l'insertion sur un territoire d'intervention défini par la conscience que les acteurs ont de sa relative homogénéité économique et sociale.

\section{- Mise en cohérence et mobilisation des acteurs}

Pour améliorer la lisibilité globale des politiques de l'emploi, plusieurs expériences de structuration de l'offre d'insertion et de mise en cohérence des politiques ont eu lieu au cours des dernières années à l'échelle du bassin d'emploi (qui apparaît comme le plus pertinent).

La DATAR a, en liaison avec le ministère du Travail et des Affaires sociales, lancé en 1995 un programme de soutien à des projets de développement des services de proximité (DATAR, 1998). Concepts flous, ces derniers sont conçus comme une réponse à des besoins nouveaux résultant des évolutions structurelles de la société. La notion de proximité qui constitue l'essence même du service rend indispensable l'analyse fine du potentiel de développement des services en fonction du territoire d'implantation et impose la mise en commun des expériences et des compétences des acteurs impliqués (communes ou groupements de communes, Etat déconcentré, ANPE, Conseils généraux, CAF, caisses de retraites et mutuelles, etc.). 
Auparavant, le Ministère du Travail avait, en mars 1994, lancé un appel au réseau des Comités de bassin d'emploi pour "favoriser l'émergence de stratégies territoriales de développement de la formation, de l'insertion et de l'emploi à l'échelle des bassins d'emploi, cohérentes et interactives avec les dispositifs nationaux et régionaux, impliquant acteurs locaux et décideurs publics et privés " (CLCBE, 1997). Le territoire est bien au centre du projet puisqu'il s'agit de construire des espaces locaux de solidarité contre l'exclusion. La constitution et l'animation des collectifs de travail sur chacun des treize sites engagés dans l'expérience y permet une vision partagée des atouts et projets pour le territoire: la démarche devient "support de la construction d'un système de valeurs identitaires collectives, qui lui donne sens et force au-delà de toutes les méthodologies, pratiques et "recettes" transférables en matière de stratégie territoriale".

\section{- Entre insertion et enfermement: les régies de quartier}

L'apport le plus important des comités de bassin d'emploi des zones rurales est d'avoir expérimenté une problématique territoriale incluant les acteurs économiques dès le démarrage des initiatives d'insertion, évitant ainsi, ou du moins limitant, la coupure habituelle entre sphère sociale et sphère productive (Wuhl, 1996). Cette coupure est par contre très forte pour les régies de quartier. Relevant du projet de développement social communautaire, toute régie de quartier s'appuie sur une volonté partenariale locale. Fondées sur une démarche d'insertion par l'économique ancrée sur un territoire défini en théorie en fonction du sentiment d'appartenance des habitants, les régies de quartier ont pour missions d'assurer une meilleure qualité des services d'entretien afin de "requalifier" le cadre de vie, de favoriser l'insertion sociale et professionnelle des habitants, de développer la vie sociale dans le quartier.

En réalité, ces territoires sont des portions d'espace urbain façonnées par les logiques administrative, politique ou patrimoniale (Office HLM) qui en ont auparavant fait des cadres de procédures de réhabilitation, des cibles de la "géographie prioritaire". L'argument du lien social et de I'ancrage communautaire (dans le quartier, avec le quartier, pour le quartier) ne renforce-t-il pas les effets de l'isolement social - au sens proposé par le sociologue W.J. Wilson pour l'urban underclass des Etats-Unis: "L'isolement social ne prive pas seulement les résidents des quartiers centraux de ressources et de modèles sociaux conventionnels, dont la présence amortissait les effets de l'absence d'emplois, mais aussi de cette sorte d'apprentissage culturel des réseaux sociaux qui facilite l'avancement économique et social dans la société industrielle moderne" (Wilson, 1991) - et $n^{\prime}$ est-il pas producteur de frontières dans la ville? L'insertion ne fonctionne pas comme un sas: les entreprises proposent des travaux de soustraitance déqualifiés qui requièrent des savoirs pratiques; les bailleurs utilisent les régies de quartiers pour entretenir et rénover à moindre coût leur patrimoine. Au total, avec ces dérives, l'insertion change radicalement de sens: au lieu d'être pensée à travers des emplois précaires mais transitoires, elle a une fonction d'adaptation durable à la précarité profes- 
sionnelle (Eme et Gruyer, 1995). Si l'insertion dans l'espace familier produit de l'enfermement qui ne permet pas la mobilité professionnelle, symbolique, relationnelle, alors elle peut, comme cela est parfois fait pour la politique de la ville, être analysée comme une politique de "cantonnement " (Delevoye, 1997).

\section{Conclusion}

Dans son bilan juridique des zones franches urbaines, Gérard Cahin conclut à l'inadaptation du critère territorial car "les effets du chômage [...] ne se cantonnent ni à certains territoires ni aux catégories de personnes servant à les définir et bénéficiant de surcroît de la clause d'embauche locale dans les ZFU. II va sans dire que les personnes répondant aux mêmes critères de définition, à l'exclusion du lieu de résidence, $n$ 'ont pas moins de titre que les premières à être aidées". L'approche géographique des questions sociales $n$ 'en est pas moins pertinente. L'espace intervient comme cadre, support, enjeu des politiques d'emploi et d'insertion: dans leur localisation, les politiques décentralisées rencontrent une inégale mobilisation des acteurs locaux; à l'amont de ces politiques, l'intensité du chômage varie avec les caractéristiques économiques et démographiques; à l'aval, elles contribuent à la production de territoires, et à ses effets sociaux.

Analyser les dimensions spatiales des politiques d'emploi et d'insertion (qui articulent le spatial, le social et le politique) revient à formuler l'une des questions qui animent la géographie sociale, celle de l'importance croissante donnée au territoire dans les politiques publiques (Séchet, 1996). Avec le traitement des problèmes sociaux par le territoire, avec la tendance, observée aussi dans les politiques de santé publique ou en matière d'école ou d'insécurité, à transformer des problèmes sociaux en problèmes urbains, est-on encore dans les logiques de compensation et de réduction des inégalités inscrites dans les espaces? Les politiques sociales, qui se placent à l'aval des processus, n'ont pas pour finalité de réduire les inégalités mais de gérer et réguler.

\section{RÉFÉRENCES}

Association des Maires de France (AMF), 1997. - Initiatives locales pour l'emploi. Rapport du groupe de travail. AUGUSTIN Jean-Pierre, PAILHÉ Joël, DUPOUEY-BORDENAVE Jeanne, BERNARD Marie-Christine, 1991. "A la recherche des dynamiques sociospatiales", in Le RMI à l'épreuve des faits. Territoires, insertion et société, Syros Alternatives, pp. 173-182.

CAHIN Gérard, 20 juin 1999. - "Les zones franches urbaines. Bilan juridique provisoire", L'Actualité juridique - Droit administratif, pp. 467-477.

CLCBE, 1997. - Expérience de mise en cohérence des politiques emploi-formation-insertion au niveau du bassin d'emploi, Rapport final présenté au Sénat le 12 décembre 1997, 63 p.

Commissariat Général du Plan, 1997. - Cohésion sociale et territoires, Rapport présidé par Jean-Paul Delevoye, La Documentation française, $218 \mathrm{p}$.

DATAR, 1998. - Activités de proximité. 43 expériences territoriales, La Documentation française, $174 \mathrm{p}$.

EME Bernard, GRUYER Bernard, 1995. - "Des territoires complexes", Territoires (Dossier "Régie de quartier: une entreprise de citoyenneté $"$ ), pp. 33-37. 
ESTIENNE Pierre, 1979. - La France. 1. Généralités. Région du Nord, Masson, Coll. Géographie des Etats, $184 \mathrm{p}$.

EYDOUX Laurence, 1995. - Chômage de longue durée et mesures d'insertion: une étude des contrats emploi-solidarité dans la région de Rennes, Mémoire de maîtrise en géographie sous la direction de Raymonde Séchet, Université Rennes 2.

GUIGOU Jean-Louis, 1995. - Une ambition pour le territoire. Aménager l'espace et le temps, DATAR, Editions de I'Aube, $136 \mathrm{p}$.

GUIGOU Jean-Louis, 1996. - “Un jeu de rôles», Pouvoirs locaux, n²9, pp. 101-104.

SOIZIG Hervé, 1996. - L'insertion des demandeurs d'emploi de longue durée et des bénéficiaires du RMI. Sa mise en place dans l'arrondissement de Fougères, mémoire de maîtrise de géographie sous la direction de Raymonde Séchet, Université Rennes 2.

JOIN-LAMBERT Marie-Thérèse, 1994. - Politiques sociales, Presses de la Fondation nationale de Sciences politiques et Dalloz, $571 \mathrm{p}$.

LE CARPENTIER J., 1992. - «Repenser la cohérence des dispositifs d'insertion. Réflexions autour du rapport du Conseil national des missions locales", in Patricia Bouillaguet et Christophe Guitton (Dir.), Le chômage de longue durée. Comprendre, agir, évaluer, Syros Alternatives, pp. 707-716.

MAUREL L., LEFĖVRE C., 1995. - "La recomposition territoriale en questions: positions d'acteurs", Revue de Géographie de Lyon, vol. 70, $\mathrm{n}^{\circ} 2$, pp. 159-168.

Ministère de l'Emploi et de la Solidarité, 1997. - Collectivités locales et lutte contre le chômage, La Documentation française, Cahier Travail et Emploi, 257 p.

MONTAGNÉ-VILLETTE Solange, 1999. - "La géographie du chômage: positionnement et objectifs" ; intervention au colloque "Faire la géographie sociale aujourd'hui"; Caen, 18-19 novembre 1999.

ROLLAND D'ERCEVILLE Soizic, 1999. - Un dispositif d'accueil des demandeurs d'emploi: les PAE en IIle-etVilaine, principes et réalités de fonctionnement, mémoire de DEA sous la direction de Raymonde Séchet.

SÉCHET Raymonde, 1996. - Espaces et pauvretés. La géographie interrogée, L'Harmattan, coll. Géographie sociale, $213 \mathrm{p}$.

SÉCHET Raymonde, LE DÉLÉZIR Ronan, 1998. - "Découper et tisser: les dispositifs d'emploi et d'insertion en Ille-et-Vilaine ", in INSEE, Les découpages du territoire, INSEE Méthodes, $n^{\circ}$ 76-77-78, pp. 313-329.

WILSON J. William, 1991. - "Public Policy Research and The Truly Disadvantaged", in Christopher Jencks et Paul Peterson (Ed.), The Urban Underclass, Washington DC, The Brookings Institution, pp. 460-481.

WUHL Simon, 1996. - Insertion: les politiques en crise, PUF, coll. Sociologie d'aujourd'hui, 285 p.

Manuscrit déposé le 1er février 2000; accepté définitivement le 5 mai 2000. 